Article

\title{
Development and Evaluation of Rifampicin Loaded Alginate-Gelatin Biocomposite Microfibers
}

\author{
Ameya Sharma ${ }^{1,2} \oplus$, Vivek Puri ${ }^{1,2}$, Pradeep Kumar ${ }^{3}{ }^{(0}$, Inderbir Singh ${ }^{1, *}$ and Kampanart Huanbutta ${ }^{4, *}$ \\ 1 Chitkara College of Pharmacy, Chitkara University, Chandigarh 140401, India; \\ ameya.sharma@chitkara.edu.in (A.S.); vivek.puri@chitkara.edu.in (V.P.) \\ 2 Chitkara University School of Pharmacy, Chitkara University, Solan 174103, India \\ 3 Wits Advanced Drug Delivery Platform Research Unit, Department of Pharmacy and Pharmacology, \\ School of Therapeutic Sciences, Faculty of Health Sciences, University of the Witwatersrand, \\ Johannesburg 2193, South Africa; pradeep.kumar@wits.ac.za \\ 4 Faculty of Pharmaceutical Sciences, Burapha University, 169, Saensook, Muang, Chonburi 20131, Thailand \\ * Correspondence: inderbir.singh@chitkara.edu.in (I.S.); kampanart@go.buu.ac.th (K.H.)
}

Citation: Sharma, A.; Puri, V.; Kumar, P.; Singh, I.; Huanbutta, K. Development and Evaluation of Rifampicin Loaded Alginate-Gelatin Biocomposite Microfibers. Polymers 2021, 13, 1514. https://doi.org/ $10.3390 /$ polym13091514

Academic Editors: PaYaM ZarrinTaj, Masoud Mozafari and Farzad Seidi

Received: 12 April 2021

Accepted: 6 May 2021

Published: 8 May 2021

Publisher's Note: MDPI stays neutral with regard to jurisdictional claims in published maps and institutional affiliations.

Copyright: (c) 2021 by the authors. Licensee MDPI, Basel, Switzerland. This article is an open access article distributed under the terms and conditions of the Creative Commons Attribution (CC BY) license (https:// creativecommons.org/licenses/by/ $4.0 /)$

\begin{abstract}
Various systematic phases such as inflammation, tissue proliferation, and phases of remodeling characterize the process of wound healing. The natural matrix system is suggested to maintain and escalate these phases, and for that, microfibers were fabricated employing naturally occurring polymers (biopolymers) such as sodium alginate, gelatin and xanthan gum, and reinforcing material such as nanoclay was selected. The fabrication of fibers was executed with the aid of extrusiongelation method. Rifampicin, an antibiotic, has been incorporated into a biopolymeric solution. RF1, RF2, RF3, RF4 and RF5 were coded as various formulation batches of microfibers. The microfibers were further characterized by different techniques such as SEM, DSC, XRD, and FTIR. Mechanical properties and physical evaluations such as entrapment efficiency, water uptake and in vitro release were also carried out to explain the comparative understanding of the formulation developed. The antimicrobial activity and whole blood clotting of fabricated fibers were additionally executed, hence they showed significant results, having excellent antimicrobial properties; they could be prominent carriers for wound healing applications.
\end{abstract}

Keywords: biopolymers; microfibers; rifampicin; alginate; gelatin; nancoclay; xanthan

\section{Introduction}

The pervaded pathogens are abolished from an impaired or disrupted tissue site for efficacious repairing [1]. To evade infection and escalate the wound healing cycle, the wound site or damaged tissue site is protected, aided by sterile dressing material [2]. The three cascades such as inflammation, proliferation and tissue remodeling complete the wound healing process [3]. The topical application of an anti-microbial drug seems to be efficacious for healing or tissue repairing [4]. Rifampicin is an antibiotic drug particularly used against Gram-positive and Gram-negative bacteria and has exemplary antibacterial properties [5]. It is a liposoluble antibiotic, so penetration into most tissues is adequate. Rifampin is an antibiotic that inhibits DNA-dependent RNA polymerase activity in susceptible cells [6]. It is a suitable antibiotic to be encapsulated in microfibers for antimicrobial activity and it could be utilized for wound healing applications as well [7]. For drug delivery applications, fibrous structures, including microfibers and nanofibers, are deemed acceptable carriers [8,9]. The microfibers are reported as an attractive option because of their flexible and soft nature. The versatile composition of fibers comprising biopolymers and reinforcing material may serve as a wound dressing material. Wound exudates are also absorbed easily by the microfibers [10]. In the present research, biocomposite biopolymeric microfibers were fabricated using alginate, gelatin, xanthan gum, and nanoclay. The microfibers were prepared employing the ionotropic gelation method, and xanthan gum [11,12] and 
nanoclay $[13,14]$ were additionally used to enhance the mechanical strength of the fibers. Furthermore, physicochemical, physico-mechanical, and morphological analyses were also executed. Within the polymeric matrix, microfibers displayed an intricate molecular interaction profile, providing an irregular drug release mechanism.

\section{Materials and Methods}

\subsection{Materials}

Sodium alginate, gelatin, calcium chloride and rifampicin were the reagents used. Rifampicin was obtained from Banson Pharmaceuticals as a gift sample. All the ingredients such as sodium alginate (CAS number-9005-38-3); xanthan gum (CAS number-1113866-2); gelatin (CAS number-9000-70-8); nanoclay (CAS number-1332-58-7) and calcium chloride (CAS number-10035-04-8) were acquired from Sigma Aldrich, St. Louis, MO, USA. In the present research analysis, all analytical grade solvents, reagents and chemicals were employed.

\subsection{Preparation of Rifampicin Loaded Biocomposite Microfibers}

For the development of rifampicin biocomposite microfibers, the ionotropic gelation method was employed, as shown in Figure 1. In brief, as shown in Table 1, initially, the polymeric solution containing sodium alginate:gelatin solutions was prepared in weight ratios (5:5). To enhance the mechanical strength, xanthan gum and nanoclay were also added. The biopolymers (alginate and gelatin) were solubilized together by stirring at $500 \mathrm{rpm}$ for $30 \mathrm{~min}$, and an aqueous biopolymeric solution was prepared in which xanthan gum and nano clay were incorporated. For complete solubilization of gelatin, the biopolymeric dispersions were heated to $50^{\circ} \mathrm{C}$. First, rifampicin $(50 \mathrm{mg})$ was dissolved in $5 \mathrm{~mL}$ of acetone and gradually incorporated into the aforementioned polymeric solution. This polymeric mixture (rifampicin + alginate + gelatin + xanthan gum + nanoclay) was then extruded into a beaker containing $1 \%$ calcium chloride (using a 22 gauge needle). The microfibers led to ionic cross-linking and were further washed with the help of water and dried (air or oven), as illustrated in Figure 1 [15-17].

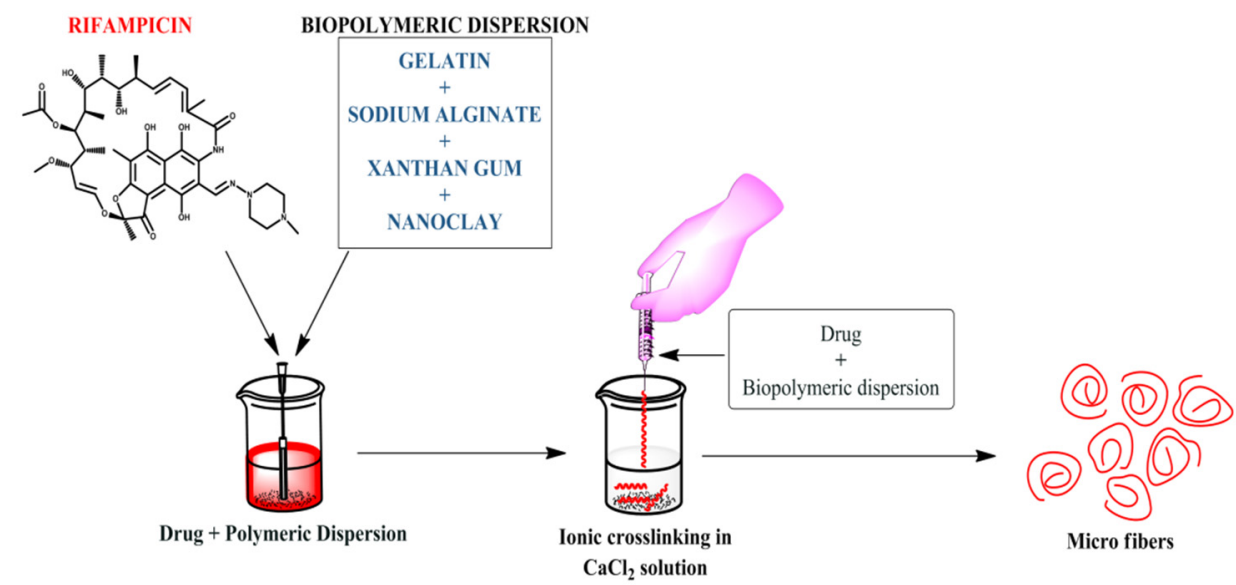

Figure 1. Fabrication method of rifampicin loaded biocomposite microfibers.

Table 1. Formulation composition of rifampicin biocomposite microfibers.

\begin{tabular}{cccccc}
\hline $\begin{array}{c}\text { Formulation } \\
\text { Batches }\end{array}$ & $\begin{array}{c}\text { Sodium Alginate } \\
\mathbf{( \% )}\end{array}$ & $\begin{array}{c}\text { Gelatin } \\
\mathbf{( \% )}\end{array}$ & $\begin{array}{c}\text { Xanthan Gum } \\
\mathbf{( \% )}\end{array}$ & $\begin{array}{c}\text { Nanoclay } \\
\mathbf{( \% )}\end{array}$ & Rifampicin (mg) \\
\hline RF1 & 2 & - & - & - & 50 \\
RF2 & 2 & 2 & - & - & 50 \\
RF3 & 2 & 2 & 0.5 & - & 50 \\
RF4 & 2 & 2 & - & 0.5 & 50 \\
RF5 & 2 & 2 & 0.5 & 0.5 & 50 \\
\hline
\end{tabular}




\subsection{Characterization of Rifampicin Loaded Biocomposite Microfibers}

The morphological characteristics of rifampicin loaded biocomposite microfibers were assessed using entrapment efficacy, SEM analysis, and physicochemical analysis through FTIR and XRD. The physical properties were also executed as epitomized water uptake properties, mechanical characteristics, in vitro release and antimicrobial properties.

\subsubsection{Entrapment Efficiency}

The cumulative amount of drug (rifampicin) which was entrapped in microfibers was calculated and weighed in ternion, employing the sample (length $10 \mathrm{~cm}$ ). To extract the maximum drug from formulated microfibers, the sample was submerged in a solution (mixture of phosphate buffer $\mathrm{pH} 7.4$ and ethanolic solvent in the ratio of 50:50 $v / v$ ) for over $24 \mathrm{~h}$. In the presence of the hydro alcoholic solvent, the microfibers were crushed in the mortar and pestle; this solution was further strained and analyzed, aiding UV-Vis analysis (Perkin Elmer Lambda35) at $337 \mathrm{~nm}$. Afterwards, a standard curve was plotted in ethanol using standard rifampicin solutions.

\subsubsection{Morphological Analysis}

In order to study morphological characteristics, the biocomposite microfibers were adhered, aided by double sided adhesive tape, and placed carefully on a metallic stub. The developed fibers were sequentially plated with gold, and under various magnifications $(250 \times$ and $500 \times)$ and at accelerating voltages $(15 \mathrm{Kv})$, the surface of the fibers was visualized. The aforementioned scanning microscopy was performed on FEI Nova Nanolab 600 SEM, and to modify the samples acquired for clarity, brightness and contrast, Paint.net (graphic editor) was used.

\subsubsection{XRD}

The X-ray diffractometry was carried out to investigate the crystallinity of rifampicin, rifampicin loaded fibers. Study was carried out using an X-ray diffractometer (Miniflex 2, Rigaku, Japan), which is a high potential and developed technique fundamentally having scattered intensity of an X-ray beam, which is hitting the sample as a function of incident and scattered angle, polarization and wavelength or energy. The flexibility and non-destructive operational protocols of the technique exhibit chemical composition, but this technique also shows the crystallographic structure of the raw samples. The

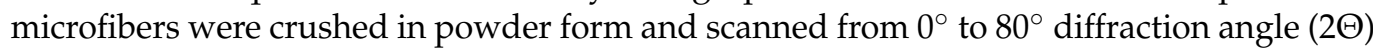
range under the following measurement conditions: source, nickel filtered $\mathrm{Cu}-\mathrm{K}$ radiation; voltage $35 \mathrm{kV}$; current $25 \mathrm{~mA}$; scan speed $0.05 \mathrm{~min}^{-1}$.

\subsubsection{DSC}

Thermal analysis was carried out employing differential scanning calorimetry with liquid nitrogen. The analysis was performed under purge of dry nitrogen gas. High purity indium was used to calibrate the heat flow and heat capacity of the instrument. Sample films of weight $(2.5-5 \mathrm{mg})$ were placed in an aluminum cell and were firmly crimped with the lid to provide an adequate seal. The sample was heated at ambient temperature from 50 to $300{ }^{\circ} \mathrm{C}$ at a preprogrammed heating rate of $10{ }^{\circ} \mathrm{C} \mathrm{min}-1$.

\subsubsection{FTIR Studies}

The spectroscopic analysis of biocomposite microfibers was performed to determine inter molecular interactions in between the drug, constituent biopolymers and reinforcing material employing (IFS66/S, Alpha Bruker, Leipzig, Germany). This study was carried out in the region of $4000-650 \mathrm{~cm}^{-1}$ wave number in the reflectance mode.

\subsubsection{Water Uptake}

The water uptake property of the biocomposite microfiber polymeric matrix was studied and calculated at $\mathrm{pH} 7.4$ phosphate buffer saline. The formulated microfibers 
were weighed (the initial weight) and then submerged for $24 \mathrm{~h}$ in the aforementioned PBS $(10 \mathrm{~mL})$ prior to being deliberately removed, aided by a tweezer or plucker. With the help of filter paper, water present on the surface was gradually soaked up and the wet weight referred to as final weight was acquired.

$\%$ Water uptake was determined using the following equation:

$$
\text { Water uptake }=\left[\frac{\text { Initial weight }- \text { Final weight }}{\text { Initial weight }}\right] \times 100
$$

\subsubsection{Mechanical Properties}

The mechanical characteristics of biocomposite microfibers were evaluated, aided by a uniaxial tensile machine. The tensile machine (Instron5943, Canton, MA, USA) was held and loaded with a cell size of $10 \mathrm{~N}$ with specified cross head speed $(5 \mathrm{~mm} / \mathrm{min})$. The biocomposite microfiber samples were sliced into a specified length of $5 \mathrm{~cm}$ and, further, this extension test was conducted at room temperature. The following equations were then used to calculate tensile strength and elongation at break.

$$
\begin{gathered}
\text { Tensile strength }=\frac{\text { Breaking force }(\mathrm{N})}{\text { cross }- \text { sec tional area of sample }\left(\mathrm{mm}^{2}\right)} \\
\text { Elongation to break }=\frac{\text { Increase in length at breaking point }(\mathrm{mm})}{\text { original length }(\mathrm{mm})} \times 100
\end{gathered}
$$

\subsubsection{In Vitro Drug Release Studies}

The drug release pattern of biocomposite microfibers was executed at $37{ }^{\circ} \mathrm{C}$ for $72 \mathrm{~h}$ in phosphate-buffered saline (PBS; $\mathrm{pH} 7.4)$. The microfibers (5 mg rifampicin) were poured into PBS $(50 \mathrm{~mL})$ in a sealed container and mounted on an orbital shaker machine. Thereafter, aliquots of 3 mLwere extracted at fixed time intervals such as $0.5,1,2,4,8,12$, 24,48 and $72 \mathrm{~h}$. All the samples were subsequently replaced with fresh PBS to perpetuate volume. The sample microfibers were removed and then analyzed at $337 \mathrm{~nm}$. To analyze the drug release mechanism, the data profile was countered into varied models such as zero order, first order, Higuchi matrix, Hixson-Crowell, and Korsmeyer-Peppas release data models-kinetic experiments were then carried out.

Zero order- $Q=Q_{0}+k_{0} t$

First order- $\ln Q=\ln Q_{0}+k_{1} t$

Higuchi model- $Q=k_{H} t^{\frac{1}{2}}$

Hixson-Crowell model- $Q_{0}^{\frac{1}{3}}-Q_{R}^{\frac{1}{3}}=k_{s} t$

Korsmeyer-Peppas model- $Q / Q_{T}=k_{k p} t^{\mathrm{n}}$

Depict:

$Q=$ total amount of drug release at time $t$;

$Q_{0}=$ initial amount of drug;

$Q_{R}=$ amount of remaining drug at time $t ;$

$Q_{T}=$ cumulative amount of drug release;

$k_{0}, k_{1}, k_{H}, k_{s}$ and $k_{k p}=$ kinetic constants for all respective models such as zero order, first order, Higuchi, Hixson-Crowell and Korsmeyer-Peppas models;

$\mathrm{n}=$ release exponent.

\subsubsection{Whole Blood Clotting}

The fibers were placed into polypropylene tubes and the aforementioned tubes were pre-warmed to $37^{\circ} \mathrm{C}$. Citrated whole blood $(0.2 \mathrm{~mL})$ was then slowly dispersed onto the fibers and then $0.2 \mathrm{M}$ of $\mathrm{CaCl}_{2}$ solution $(20 \mu \mathrm{L})$ added to start coagulation. The tubes containing blood samples were incubated at $37^{\circ} \mathrm{C}+30 \mathrm{rpm}$ for $10 \mathrm{~min}$. After $10 \mathrm{~min}$, red blood cells that are free and are not trapped in the clot were hemolyzed with $25 \mathrm{~mL}$ of water and the absorbance of the resulting hemoglobin solution was measured at $540 \mathrm{~nm}$. 


\subsubsection{Antimicrobial Studies}

The agar disc method was employed in order to assess the antimicrobial activity of the formulated biocomposite fibers. The two bacterial strains such as Staphylococcus aureus (Gram-positive bacterium) and Escherichia coli (Gram-negative bacterium), primarily present in the wound bed, were used in this process. The solution for agar was developed using the technique using Hi-Media. Briefly, $28 \mathrm{~g}$ of agar powder was dispersed in $1000 \mathrm{~mL}$ of filtered water. The solution was heated to boil so that the medium was fully dissolved and further sterilized by autoclaving at $121^{\circ} \mathrm{C}$ for $15 \mathrm{~min}$. Cooling of up to $40-50{ }^{\circ} \mathrm{C}$ was allowed, and by pouring $20 \mathrm{~mL}$ of liquid agar media, the agar plates were prepared. The bacterial culture suspension of S. aureus and E. coli was inoculated in large Petri dishes to initiate rapid growth of microorganisms. To become solidified, Petri plates were retained and a $6 \mathrm{~mm}$ pit was created, plying a sterilized borer. The drug-containing control group (rifampicin) and rifampicin fiber (RF5) were mounted in an agar plate pit and incubated for $24 \mathrm{~h}$ at $37^{\circ} \mathrm{C}$, with a deliberate inhibition zone.

\section{Results and Discussion}

The entrapment efficiency of rifampicin loaded microfibers was found to be 91.14 $\pm 3.23 \%$ for RF1 and $96.34 \pm 1.76 \%$ for RF5 microfibers, as seen in Figure 5 and Table 2 . Contrastingly, the entrapment efficiency was improved with an inclusion of gelatin in microfibers, which could be attributed to partial inter molecular interactions between the drug (rifampicin) and gelatin. RF3's entrapping efficiency was $95 \pm 2.31 \%$, which may be attributed to xanthan gum incorporation. The microfibers' (RF5) entrapment efficiency was found to be the highest because of the addition of xanthan gum and nanoclay, which may be due to enriched interaction between the polymeric molecules and reinforcing material. SEM was used to study the surface morphology of alginate/gelatin biocomposite microfibers. Between 200 and $400 \mu \mathrm{m}$, the diameter of the microfibers was determined. On the surface of the microfibers, crystal deposition was visualized clearly, which could be attributable due to the precipitation of calcium chloride crystals after deposition to the surface during the process of drying. A smooth homogeneous morphology was revealed on the surface that clearly demonstrated augmented miscibility and consistent homogeneity between the polymers, as shown in Figure 2.

Rifampicin has a crystalline nature and exhibits major peaks at $2 \theta=11.1,12.7,15.8$, $16.3,17.2,18.1$, and 20, respectively, according to the effects of the XRD pattern. In fabricated microfiber, the loss of crystalline peaks was observed (RF5). The results showed intercalation and potential bonding between the polymers, thus indicating that the drug was effectively trapped between the polymeric matrix in the microfibers, as depicted in Figure 3a. To scrutinize the interaction of polymers with the crystalline nature of entrapped rifampicin in the polymeric matrix of microfibers, and further embedded into the reservoir system of transdermal films, DSC analysis was performed and DSC curves are depicted in Figure $3 \mathrm{~b}$. The thermogram suggested that rifampicin showed an endothermic sharp peak at $183.7^{\circ} \mathrm{C}$ whereas this prominent peak was found to be absent, which confirmed that the drug was molecularly dispersed into the matrix of rifampicin microfibers. It also confirmed its existence in an amorphous form.

Table 2. Various parameters of formulation batches.

\begin{tabular}{ccccc}
\hline $\begin{array}{c}\text { Formulation } \\
\text { Code }\end{array}$ & $\begin{array}{c}\text { Entrapment } \\
\text { Efficiency (\%) }\end{array}$ & $\begin{array}{c}\text { Water Uptake } \\
\mathbf{( \% )}\end{array}$ & $\begin{array}{c}\text { Tensile } \\
\text { Strength } \\
\text { (N/mm } \mathbf{~})\end{array}$ & $\begin{array}{c}\text { Elongation to } \\
\text { Break (\%) }\end{array}$ \\
\hline RF1 & $91.14 \pm 3.23$ & $36.42 \pm 1.22$ & $7.12 \pm 0.25$ & $15.20 \pm 0.98$ \\
RF2 & $94.34 \pm 1.24$ & $38.16 \pm 2.18$ & $9.44 \pm 0.73$ & $17.46 \pm 0.42$ \\
RF3 & $95.21 \pm 2.31$ & $47.11 \pm 2.14$ & $19.23 \pm 0.72$ & $32.27 \pm 1.06$ \\
RF4 & $94.97 \pm 2.17$ & $42.52 \pm 2.64$ & $18.21 \pm 0.78$ & $33.34 \pm 1.07$ \\
RF5 & $96.34 \pm 1.76$ & $47.24 \pm 3.28$ & $25.28 \pm 0.76$ & $42.09 \pm 1.09$ \\
\hline
\end{tabular}




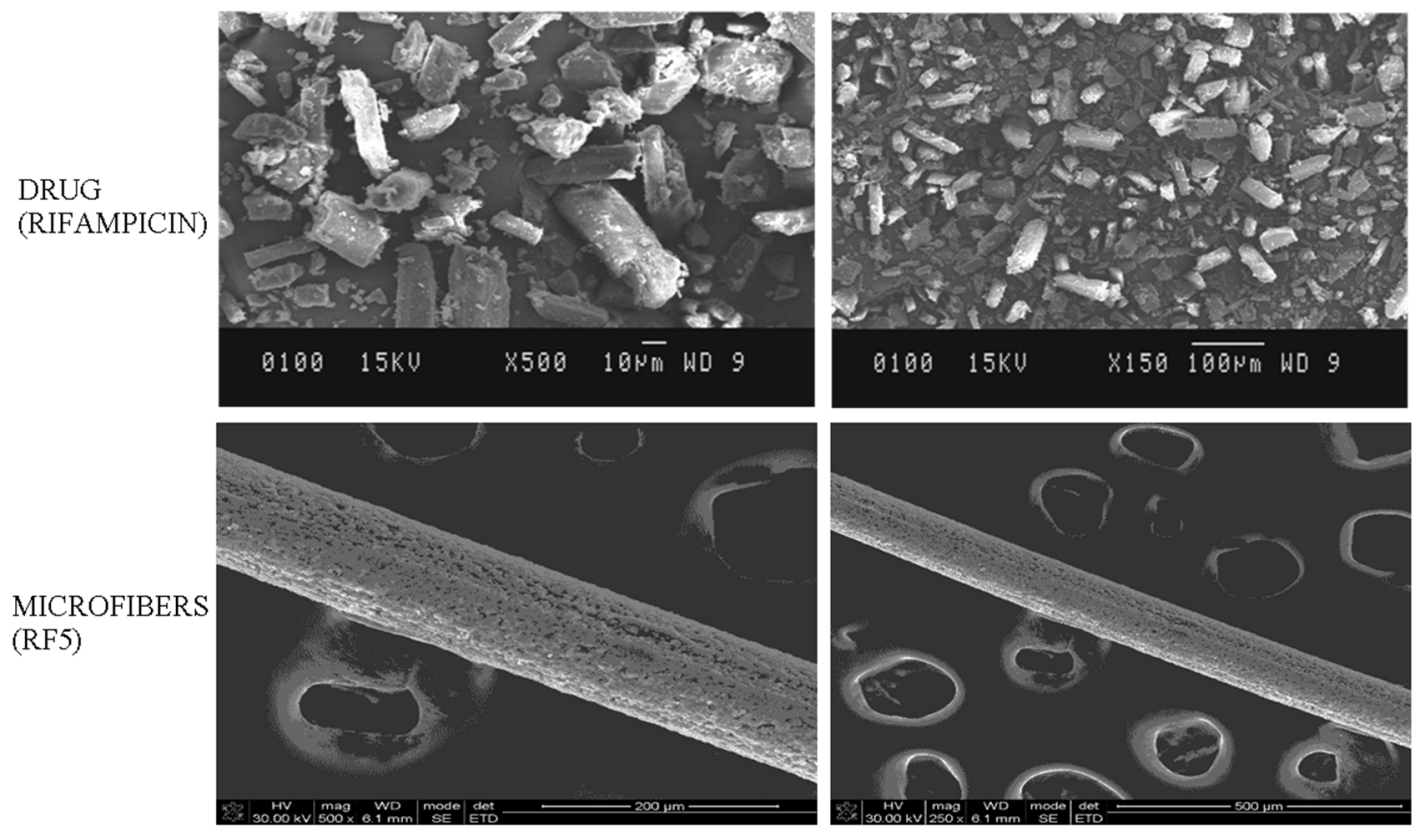

Figure 2. Morphological analysis of drug (rifampicin) and microfibers (RF5) at magnification value at $500 \times$ and $150 \times$.
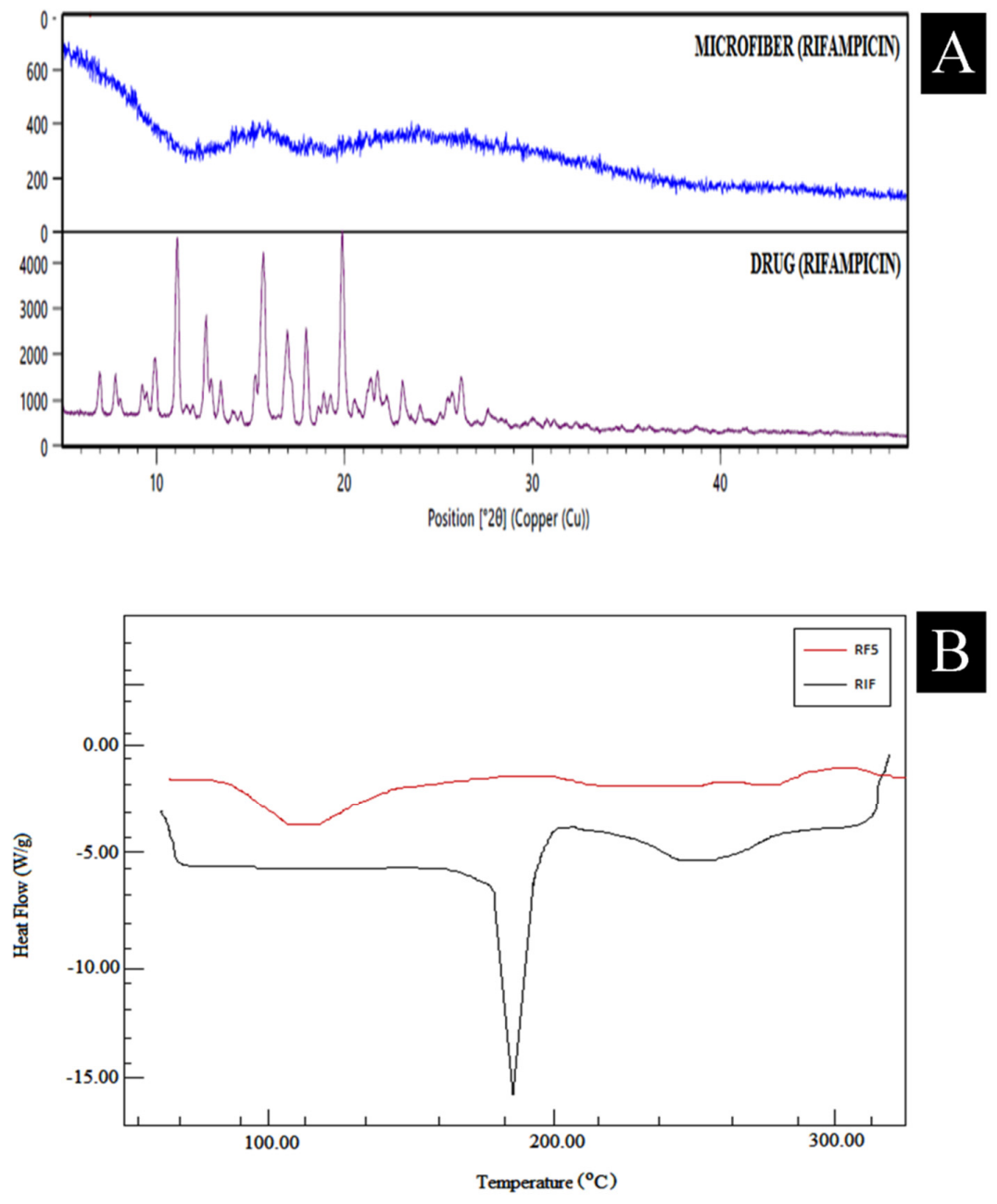

Figure 3. (A) XRD spectra of drug (rifampicin) and microfiber (RF5), (B) DSC thermogram of drug (rifampicin) and microfiber (RF5). 
In order to provide accurate information on the interaction seen between formulations, Fourier transform infrared (FTIR) attenuated total reflectance was used. The FTIR spectra of the drug (rifampicin), formulated rifampicin microfibers and polymers used are shown in Figure 4. The FTIR drug rifampicin spectrum showed a peak of $3472 \mathrm{~cm}^{-1}$ (NH stretching), $2894 \mathrm{~cm}^{-1}$ (C-H bonding), $1629 \mathrm{~cm}^{-1}(\mathrm{C}=\mathrm{O}), 1478 \mathrm{~cm}^{-1}(\mathrm{C}=\mathrm{C}), 1379 \mathrm{~cm}^{-1}(\mathrm{CH} 2, \mathrm{C}=\mathrm{C})$, $1059 \mathrm{~cm}^{-1}(-\mathrm{CH}, \mathrm{CO}, \mathrm{C}-\mathrm{H})$, and $987 \mathrm{~cm}^{-1}$ (三C-H, C-H) [18]. The IR range of alginate had characteristic absorption peaks at $3311 \mathrm{~cm}^{-1}$, which showed $\mathrm{OH}$ stretching, $1612 \mathrm{~cm}^{-1}$, which showed carboxylic $\mathrm{C}=\mathrm{O}, 1298 \mathrm{~cm}^{-1}$, which possessed $\mathrm{C}-\mathrm{CH}, 1086$ and $1059 \mathrm{~cm}^{-1}$, ascribed to C-O stretching, $1032 \mathrm{~cm}^{-1}$, which represented C-C, $942 \mathrm{~cm}^{-1}$, which showed $\mathrm{C}-\mathrm{O}, 888 \mathrm{~cm}^{-1}$, which manifested $\mathrm{CH}$, and $822 \mathrm{~cm}^{-1}$, which represented Na-O [19]. The substantial gelatin peaks showed absorption bands at $3414 \mathrm{~cm}^{-1}$, confirmed NH stretching, $1655 \mathrm{~cm}^{-1}$, confirmed amide $\mathrm{C}=\mathrm{O}, 1556 \mathrm{~cm}^{-1}$, proclaimed amide $\mathrm{NH}$ bending, and $1343 \mathrm{~cm}^{-1}$, ascribed to C-N stretching [20]. The prominent peaks of xanthan gum showed peaks at $3427 \mathrm{~cm}^{-1}$ (O-H stretching), $2995 \mathrm{~cm}^{-1}$ (aliphatic C-H stretching), $1635 \mathrm{~cm}^{-1}$ (COO symmetric stretching), $1434 \mathrm{~cm}^{-1}$ (asymmetric stretching) and $1245-1043 \mathrm{~cm}^{-1}$ (pyranoid C-O-C ring stretching) [21]. The FTIR spectra of nanoclay exhibited peaks at $3442 \mathrm{~cm}^{-1}$ (OH stretching), $2916-2846 \mathrm{~cm}^{-1}$ (methylene symmetric vibration), $1737-1467 \mathrm{~cm}^{-1}$ (C=O, C-O-H stretching and hydrogen bonds), and $1063 \mathrm{~cm}^{-1}$ (Si-O bending) [22]. The FTIR spectrum relating to microfibers was similar to the polymer spectrum. Due to the lack of optimum peak characteristics of rifampicin in the FTIR spectra of microfibers as they have been cloaked by bands formed by polymers, it could be inferred that rifampicin was well embedded in the microfibers [17].

In developed microfibers, the water uptake property or capability of alginate-gelatin biocomposite microfibers was found to be enhanced; improved network formation was as imparted by gelatin concentration. In addition to xanthan gum and nanoclay, the percentage of water absorption of biocomposite microfibers was decreased, with a maximum value of $47.24 \pm 3.28 \%$ in the case of RF5 (Figure 5 and Table 2). As rifampicin is a lipophilic drug, its prevalence in the biopolymer matrix may be due to this reduction in water affinity.

Pertaining to mechanical parameters, tensile strength and elongation to break (extensibility) were evaluated. Tensile strength and break elongation differed between $7.12 \pm 0.25$ to $25.28 \pm 0.76 \mathrm{~N} / \mathrm{mm}^{2}$ and $15.20 \pm 0.98 \%$ to $42.09 \pm 1.09 \%$. With the addition of xanthan gum and nanoclay, the tensile strength of the microfibers improved as illustrated in Figure 5 and Table 2. An increase in the mechanical strength of biocomposite fibers may be due to the association and bond formation between the biopolymers used. The addition of nanoclay has significantly enhanced this mechanical property. This may be due to the reinforcing material's capability to act as fillers (rigid) in the polymeric matrix, as well as intermolecular interactions between the polymeric matrix and layered silicates through $\mathrm{H}$ bonds. As explained in the FTIR discussion, the tensile strength in blended microfibers is effectively enhanced due to the partial contact between the biomacromolecules [20].

The results of the in vitro drug release study revealed that microfibers containing a combination of polymeric content and reinforcing material resulted and thereby minimized the total percent release of the drug. The drug releases from RF1, RF2, RF3, RF4 and RF5 were observed to be $31.28 \pm 4.36 \%, 20.23 \pm 3.12 \%, 14.06 \pm 2.2 \%, 17.22 \pm 3.08 \%$ and $9.03 \pm 2.04 \%$, respectively, in $90 \mathrm{~h}$, as depicted in Figure 6. Alginate polymer matrix formation may be a retardant factor in the release of the drug from prepared fibers. Furthermore, the incorporation of xanthan gum and nanoclay delayed drug release from prepared microfibers. This may be due to the formation of intermolecular hydrogen bonds between polymeric chains and reinforcing material in biocomposite fibers. Molecular interaction with polymeric chain relaxation (up to hydration) could also lead to a decrease in the release of the drug from RF5. The drug release data obtained after in vitro release study were further analyzed via fitting into various kinetic models, i.e., zero order, first order, Higuchi equation, Hixson-Crowell model, and the power law. The coefficient of determination $\left(r^{2}\right)$ was much closer to 1 for the first order equation. All the formulations followed non-Fickian diffusion with $n$ values falling within the $0.5<n<1.0$ range and depicted 
anomalous release behavior as shown in Table 3. This value indicates a combination of diffusion and erosion controlled mechanisms responsible for the release of the drug from the biocomposite fibers.

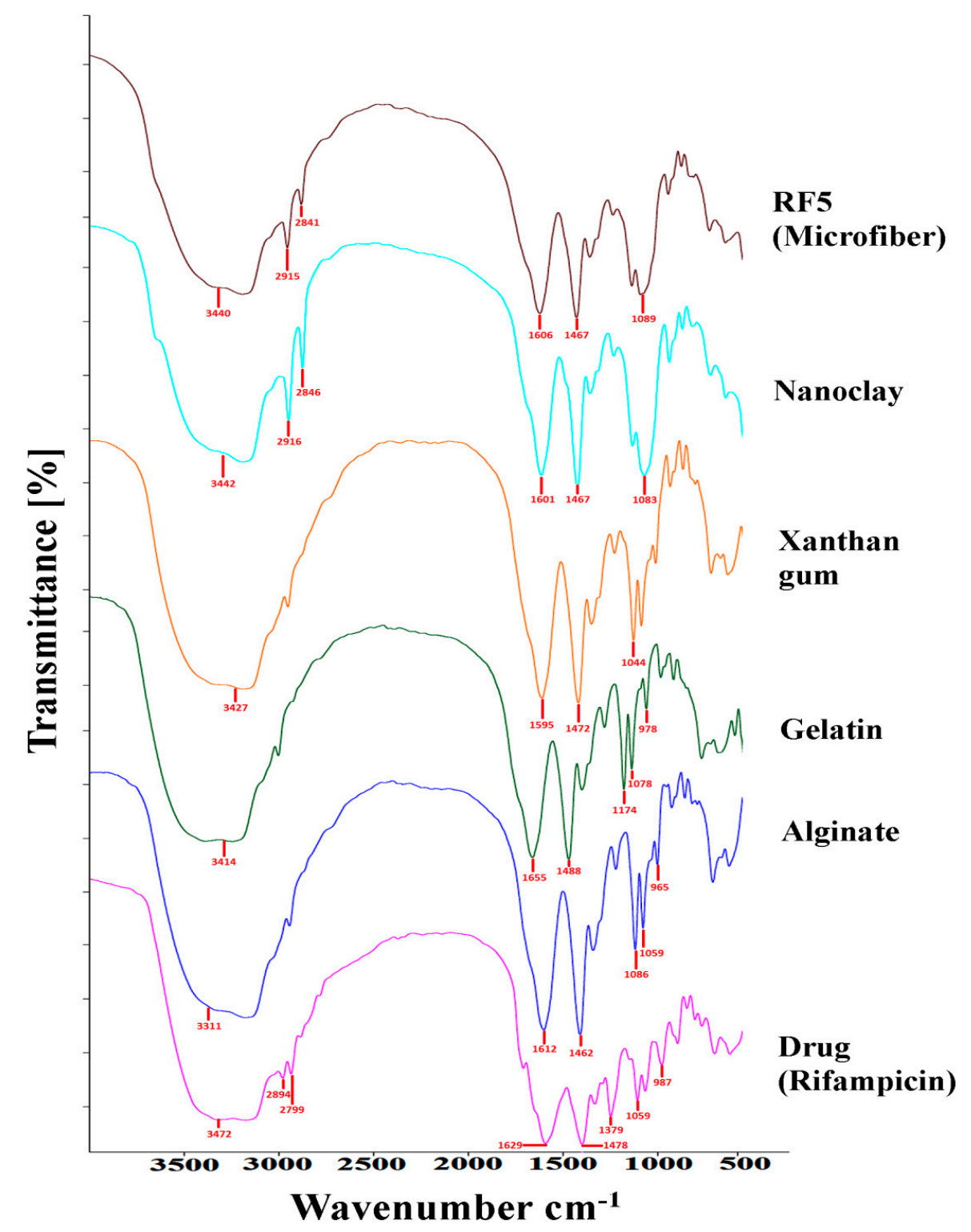

Figure 4. FTIR spectra of drug (rifampicin), alginate, gelatin, nanoclay and microfiber (RF5). 

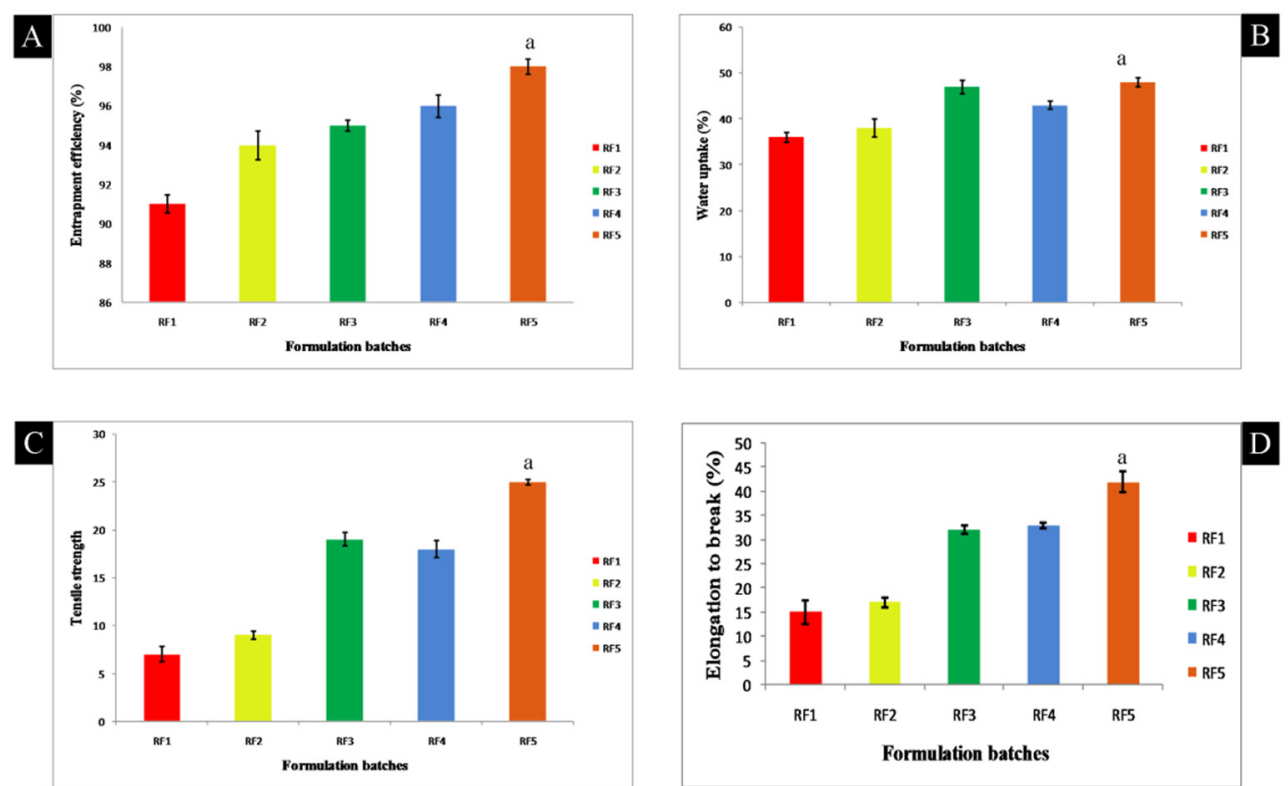

Figure 5. Bar graphs of different parameters (A) entrapment efficiency; (B) water uptake; (C) tensile strength (D) elongation to break (data were presented as mean \pm SD and analyzed by one-way ANOVA followed by Tukey's test as post hoc analysis. 'a' represents $p<0.05$ vs. RF1, RF2, RF3 and RF4).

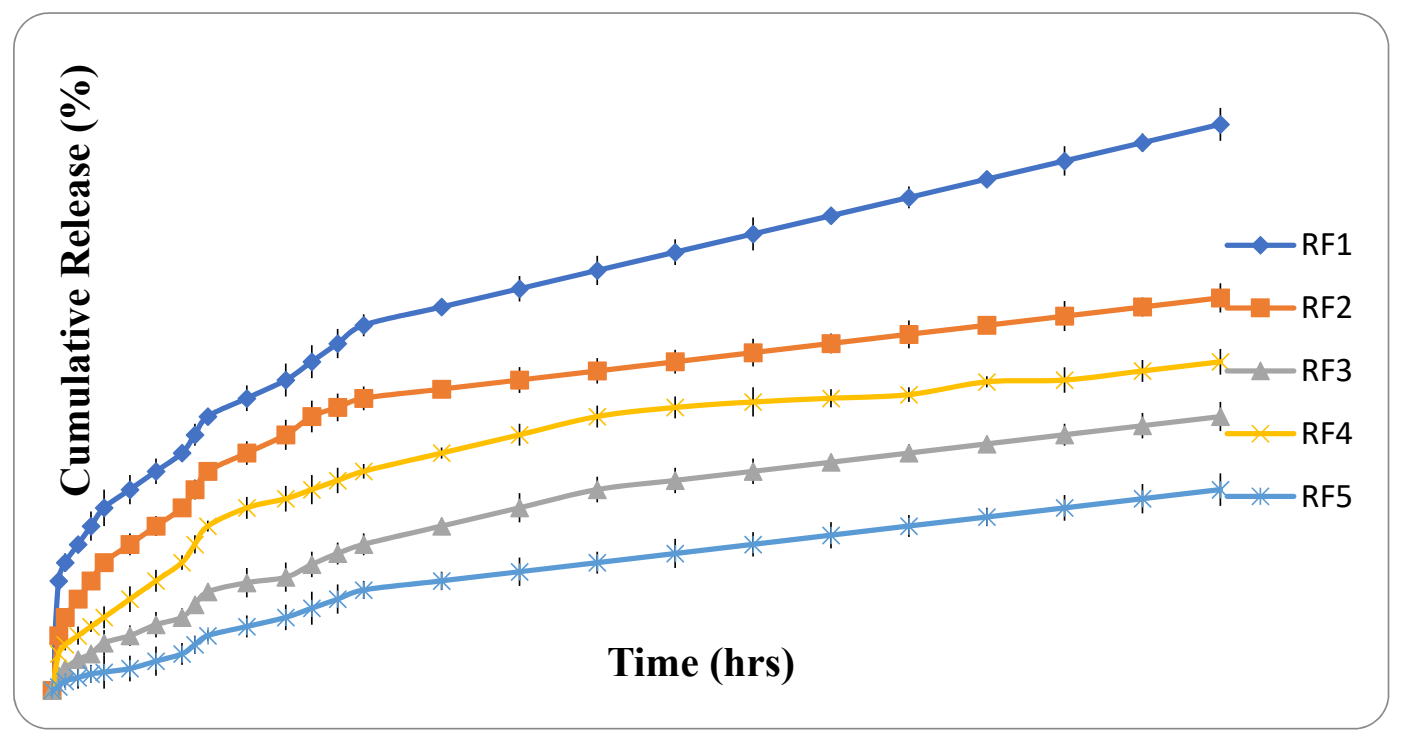

Figure 6. In vitro release of various formulation batches of microfibers.

Table 3. Drug release kinetic modeling data of various microfiber batches.

\begin{tabular}{|c|c|c|c|c|c|c|c|c|c|c|c|}
\hline \multirow{2}{*}{ Batches } & \multicolumn{2}{|c|}{ Zero Order } & \multicolumn{2}{|c|}{ First Order } & \multicolumn{2}{|c|}{ Higuchi Model } & \multicolumn{2}{|c|}{$\begin{array}{c}\text { Hixson Crowell } \\
\text { Model }\end{array}$} & \multicolumn{3}{|c|}{ KorsmeyerPeppas Model } \\
\hline & $r^{2}$ & $k_{\mathrm{o}}$ & $r^{2}$ & $k_{1}$ & $r^{2}$ & $k_{\mathrm{H}}$ & $r^{2}$ & $k_{\mathrm{HC}}$ & $r^{2}$ & $K_{\mathrm{kp}}$ & $N$ \\
\hline RF1 & 0.877 & 0.271 & 0.980 & -0.012 & 0.984 & 2.922 & 0.972 & -0.020 & 0.988 & 0.817 & 0.334 \\
\hline RF2 & 0.780 & 0.190 & 0.970 & -0.014 & 0.942 & 2.131 & 0.939 & -0.019 & 0.981 & 0.616 & 0.388 \\
\hline RF3 & 0.928 & 0.160 & 0.992 & -0.013 & 0.944 & 1.683 & 0.993 & -0.018 & 0.994 & 0.061 & 0.392 \\
\hline RF4 & 0.832 & 0.177 & 0.989 & -0.015 & 0.965 & 1.947 & 0.965 & -0.020 & 0.977 & 0.388 & 0.424 \\
\hline RF5 & 0.951 & 0.121 & 0.983 & -0.011 & 0.986 & 1.254 & 0.988 & -0.015 & 0.985 & 0.403 & 0.469 \\
\hline
\end{tabular}


In order to evaluate whether blank fibers and rifampicin loaded fibers (RF5) can increase the rate of blood clotting, whole blood was contacted with fibers for $10 \mathrm{~min}$ before hemolyzing RBCs that were not trapped in the clot that formed on the fiber surface. A higher absorbance value of the hemoglobin solution thus indicates a slower clotting rate. Rifampicin loaded fibers led to significantly lower absorbance values than blank fibers, as shown in Figure 7a. The antimicrobial activity of rifampicin against bacteria (Gram-positive and Gram-negative bacteria) was assessed using the disc diffusion method. The zone of inhibition of microfiber (RF5) was found to be $23 \mathrm{~mm}$ against $S$. aureus and $22 \mathrm{~mm}$ against E. coli. In comparison to the normal or control group, rifampicin had an inhibition zone of $24 \mathrm{~mm}$ against $S$. aureus and $20 \mathrm{~mm}$ against $E$. coli. The antimicrobial study demonstrated that the drug was effectively released from the microfiber polymer matrix and exhibited antimicrobial activity, which can be seen in Figure $7 \mathrm{~b}$.
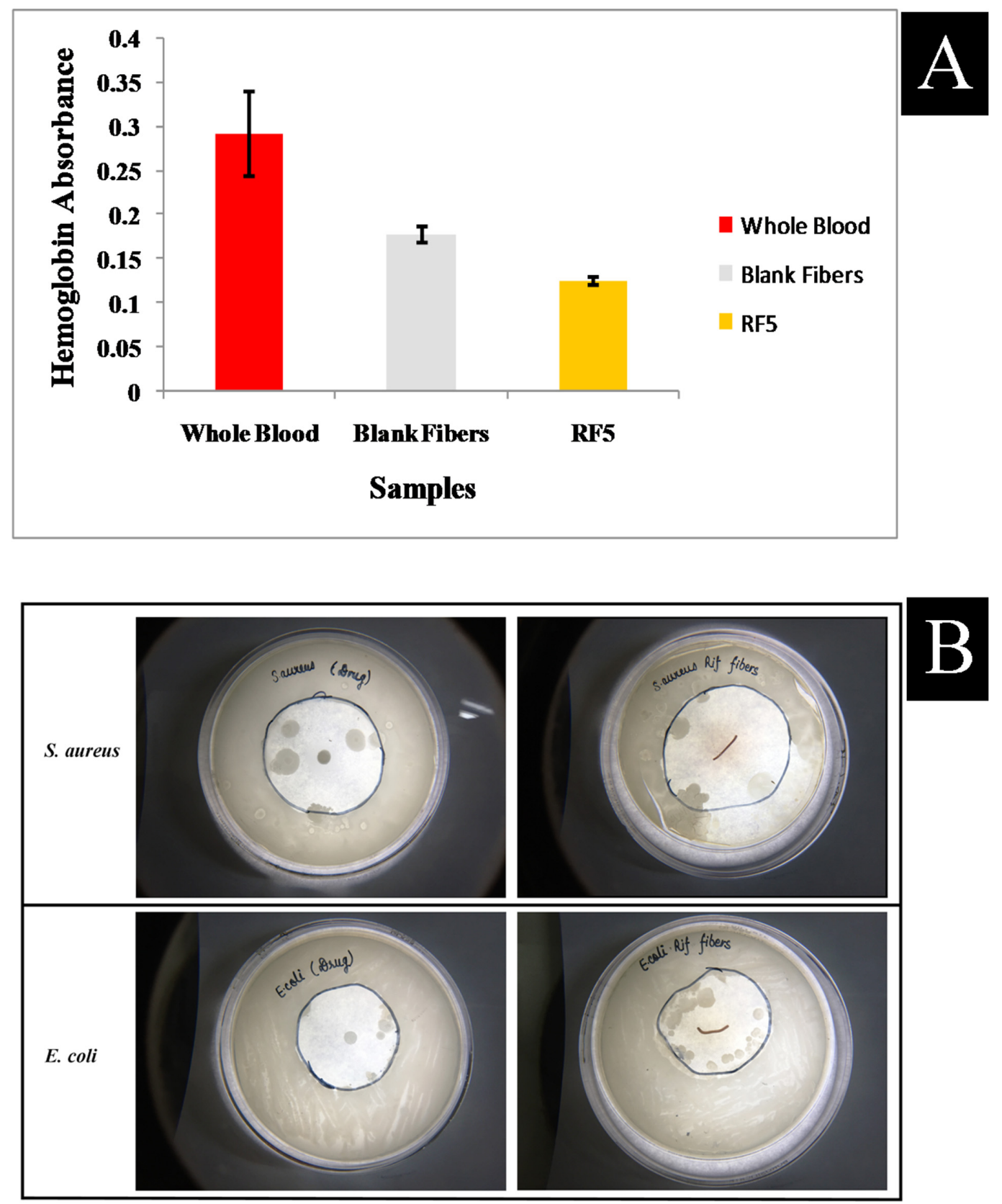

Figure 7. (A) Whole blood clotting of whole blood, blank microfibers and rifampicin loaded microfibers, (B) Antimicrobial activity of standard group (Rifampicin) and microfibers (RF5) against Staphylococcus aureus and Escherichia coli. 


\section{Conclusions}

An appropriate analytical method for rifampicin was developed by the UV/Visible spectrophotometer, and rifampicin showed maximal absorption at a wavelength of $337 \mathrm{~nm}$ in a solution mixture comprised of phosphate buffer saline $(\mathrm{pH} 7.4)$ and ethanol in the ratio $(50: 50, v / v)$. The formulation produced was identified by different techniques such as SEM, XRD, DSC and FTIR. The addition of xanthan gum and nanoclay relatively enhanced the mechanical strength individually, and when used in the combination with alginate and gelatin, it significantly increased the mechanical properties. The kinetic analysis was conducted and fitted into various kinetic models in which the anomalous behavior of all formulations produced was represented by $\mathrm{n}$ value. As a controlled drug delivery system, the formulation batches have maximum release at $90 \mathrm{~h}$, implying the prolonged retardation of drug release from the prepared microfibers. Since biocomposite microfibers have good mechanical properties, they may be an effective candidate for developing a controlled drug delivery system and prolonged drug delivery system. The microfibers could be potential candidates and effective biopolymeric wound dressing materials for wound healing applications, as they implied excellent anti-microbial properties.

Author Contributions: I.S., K.H. and P.K. conceived and designed the experiments, A.S. performed the experiments, V.P. contributed reagents/materials/analyses tools and A.S. wrote the paper. All authors have read and agreed to the published version of the manuscript.

Funding: The authors received no external funding.

Institutional Review Board Statement: Not applicable.

Informed Consent Statement: Not applicable.

Data Availability Statement: Not applicable.

Acknowledgments: The authors thankfully acknowledge the support and institutional facilities provided by Chitkara College of Pharmacy, Chitkara University, Punjab, India.

Conflicts of Interest: The authors declare no conflict of interest.

\section{References}

1. Bhardwaj, N.; Chouhan, D.; B Mandal, B. Tissue engineered skin and wound healing: Current strategies and future directions. Curr. Pharm. Des. 2017, 23, 3455-3482. [CrossRef]

2. Sharma, A.; Puri, V.; Kumar, P.; Singh, I. Biopolymeric, nanopatterned, fibrous carriers for wound healing applications. Curr. Pharm. Des. 2020, 26, 4894-4908. [CrossRef]

3. Manzini, B.M.; Machado, L.M.R.; Noritomi, P.Y.; da Silva, J.V.L. Advances in Bone tissue engineering: A fundamental review. J. Biosci. 2021, 46, 1-18. [CrossRef]

4. Mazini, L.; Rochette, L.; Hamdan, Y.; Malka, G. Skin Immunomodulation during Regeneration: Emerging New Targets. J. Pers. Med. 2021, 11, 85. [CrossRef]

5. Pyta, K.; Przybylski, P.; Klich, K.; Stefańska, J. A new model of binding of rifampicin and its amino analogues as zwitterions to bacterial RNA polymerase. Org. Biomol. Chem. 2012, 10, 8283-8297. [CrossRef] [PubMed]

6. Sharma, A.; Puri, V.; Kumar, P.; Singh, I. Rifampicin-Loaded Alginate-Gelatin Fibers Incorporated within Transdermal Films as a Fiber-in-Film System for Wound Healing Applications. Membranes 2021, 11, 7. [CrossRef]

7. Gilchrist, S.E.; Lange, D.; Letchford, K.; Bach, H.; Fazli, L.; Burt, H.M. Fusidic acid and rifampicin co-loaded PLGA nanofibers for the prevention of orthopedic implant associated infections. J. Control. Release 2013, 170, 64-73. [CrossRef] [PubMed]

8. Vicosa, A.L.; Gomes, A.C.O.; Soares, B.G.; Paranhos, C.M. Effect of sepiolite on the physical properties and swelling behavior of rifampicin-loaded nanocomposite hydrogels. Express Polym. Lett. 2009, 3, 518-524. [CrossRef]

9. Nathanael, A.J.; Oh, T.H. Biopolymer Coatings for Biomedical Applications. Polymers 2020, 12, 3061. [CrossRef]

10. Álvarez-Suárez, A.S.; Dastager, S.G.; Bogdanchikova, N.; Grande, D.; Pestryakov, A.; García-Ramos, J.C.; Villarreal-Gómez, L.J. Electrospun fibers and sorbents as a possible basis for effective biocomposite wound dressings. Micromachines 2020, $11,441$. [CrossRef] [PubMed]

11. Hazirah, M.N.; Isa, M.I.N.; Sarbon, N.M. Effect of xanthan gum on the physical and mechanical properties of gelatincarboxymethyl cellulose film blends. Food Packag. Shelf Life 2016, 9, 55-63. [CrossRef]

12. Alves, A.; Miguel, S.P.; Araujo, A.R.; de Jesús Valle, M.J.; Sánchez Navarro, A.; Correia, I.J.; Coutinho, P. Xanthan gum-Konjac glucomannan blend hydrogel for wound healing. Polymers 2020, 12, 99. [CrossRef] [PubMed] 
13. Noori, S.; Kokabi, M.; Hassan, Z.M. Nanoclay enhanced the mechanical properties of poly (vinyl alcohol)/chitosan/montmorillonite nanocomposite hydrogel as wound dressing. Procedia Mater. Sci. 2015, 11, 152-156. [CrossRef]

14. Tian, H.; Wang, K.; Liu, D.; Yan, J.; Xiang, A.; Rajulu, A.V. Enhanced mechanical and thermal properties of poly (vinyl alcohol)/corn starch blends by nanoclay intercalation. Int. J. Biol. Macromol. 2017, 101, 314-320. [CrossRef]

15. Bediako, J.K.; Lin, S.; Sarkar, A.K.; Zhao, Y.; Choi, J.W.; Song, M.H.; Yun, Y.S. Benignly-fabricated crosslinked polyethylenimine/calciumalginate fibers as high-performance adsorbents for effective recovery of gold. J. Clean. Prod. 2020, 252, 119389. [CrossRef]

16. Singh, P.; Singh, S.K.; Bajpai, J.; Bajpai, A.K.; Shrivastava, R.B. Iron crosslinked alginate as novel nanosorbents for removal of arsenic ions and bacteriological contamination from water. J. Mater Res. Tech. 2014, 3, 195-202. [CrossRef]

17. Sharma, A.; Mittal, A.; Puri, V.; Kumar, P.; Singh, I. Curcumin-loaded, alginate-gelatin composite fibers for wound healing applications. 3 Biotech 2020, 10, 1-13. [CrossRef]

18. Motiei, M.; Pleno de Gouveia, L.; Sopík, T.; Vícha, R.; Skoda, D.; Císar, J.; Khalili, R.; Domincová Bergerova, E.; Munster, L.; Fei, H.; et al. Nanoparticle-Based Rifampicin Delivery System Development. Molecules 2021, 26, 2067. [CrossRef]

19. Varaprasad, K.; Nunez, D.; Ide, W.; Jayaramudu, T.; Sadiku, E.R. Development of high alginate comprised hydrogels for removal of $\mathrm{Pb}$ (II) ions. J. Mol. Liquids 2020, 298, 112087. [CrossRef]

20. Mamidi, N.; Romo, I.L.; Leija Gutierrez, H.M.; Barrera, E.V.; Elías-Zúñiga, A. Development of forcespun fiber-aligned scaffolds from gelatin-zein composites for potential use in tissue engineering and drug release. MRS Commun. 2018, 8, 885-892. [CrossRef]

21. Nejadmansouri, M.; Razmjooei, M.; Safdarianghomsheh, R.; Shad, E.; Delvigne, F.; Khalesi, M. Semi-continuous production of xanthan in biofilm reactor using Xanthomonas campestris. J. Biotechnol. 2021, 328, 1-11. [CrossRef] [PubMed]

22. Chan, M.L.; Lau, K.T.; Wong, T.T.; Cardona, F. Interfacial bonding characteristic of nanoclay/polymer composites. Appl. Surf. Sci. 2011, 258, 860-864. [CrossRef] 